ARTICLE

https://doi.org/10.1038/s41467-020-14435-5

\title{
Radical-mediated C-C cleavage of unstrained cycloketones and DFT study for unusual regioselectivity
}

Mingyang Wang ${ }^{1,3}$, Man $\mathrm{Li}^{2,3}$, Shan Yang ${ }^{1,3}$, Xiao-Song Xue (iD ${ }^{2 \star}$, Xinxin Wu ${ }^{1} \&$ Chen Zhu (iD ${ }^{1 \star}$

The C-C $\sigma$-bond activation of unstrained cycloketones represents an ingenious and advanced technique in synthetic chemistry, but it remains a challenging area which has been largely underexplored. Herein we report an efficient strategy for the direct C-C cleavage of cyclohexanones and cyclopentanones. The cyclic $\mathrm{C}-\mathrm{C} \sigma$-bond is readily cleaved under mild conditions with the aid of an in situ formed side-chain aryl radical. Density functional theory calculations are carried out to shed light on the unusual regioselectivity of $\mathrm{C}-\mathrm{C}$ bond cleavage. The reaction affords a variety of structurally diverse 3-coumaranones and indanones that widely exist in natural products and bioactive molecules, illustrating the synthetic value of this method.

\footnotetext{
${ }^{1}$ Key Laboratory of Organic Synthesis of Jiangsu Province, College of Chemistry, Chemical Engineering and Materials Science, Soochow University, 199 RenAi Road, 215123 Suzhou, Jiangsu, China. ${ }^{2}$ State Key Laboratory of Elemento-organic Chemistry, College of Chemistry, Nankai University, 300071

Tianjin, China. ${ }^{3}$ These authors contributed equally: Mingyang Wang, Man Li, Shan Yang. *email: xuexs@nankai.edu.cn; chzhu@suda.edu.cn
} 
T he $\mathrm{C}-\mathrm{C} \sigma$-bond is ubiquitous that constitutes the framework of organic compounds; therefore, the direct elaboration of $\mathrm{C}-\mathrm{C} \sigma$-bond into other valuable chemical bonds represents an ideal, atom- and step-economic synthetic tactic $^{1-9}$. However, C-C $\sigma$-bonds usually remain inert in the transition metal-catalyzed reactions attributed to the poor interaction between the catalytic metal center and the $\mathrm{C}-\mathrm{C} \sigma$-orbital induced by the steric congestion and highly oriented nature of $\mathrm{C}-\mathrm{C} \sigma$-bonds ${ }^{10}$. Taking advantage of ring-strain relief, over the past few decades, small-sized rings such as cyclopropane and cyclobutane derivatives have served as privileged precursors in the transition metal-catalyzed $\mathrm{C}-\mathrm{C}$ activation ${ }^{11-20}$. This feature has also been applied to the alkoxy-radical-promoted $\beta-C-C$ bond scission that offers an efficient pathway to cleave the cyclic $\mathrm{C}-\mathrm{C} \sigma$-bonds of cyclopropanols and cyclobutanols ${ }^{21-27}$. In contrast, the $\mathrm{C}-\mathrm{C}$ activation of unstrained cyclic skeletons, particularly five- and six-membered rings, is still confronted with a formidable challenge ${ }^{28-31}$.

As fundamental structural units, cyclohexanone and cyclopentanone are readily available in numerous natural products and fine chemicals. With the exception of classic ring-expansion reactions such as the Bayer-Villiger oxidation and Schmidt reaction, however, the $\mathrm{C}-\mathrm{C}$ activation of cyclohexanone and cyclopentanone has been rarely explored due to the significantly decreased ring-strain energy and remarkable stability (Fig. 1a) ${ }^{32}$. Recently, Dong et al. developed an elegant strategy based on the installation of temporary directing group to activate the $\mathrm{C}-\mathrm{C}$ bond of cyclopentanones through transition metal-catalysis (Fig. 1b) ${ }^{33-35}$. But the strategy was less efficient for $\mathrm{C}-\mathrm{C}$ activation of the relatively more stable cyclohexanones. Herein, we disclose an efficient strategy for the direct C-C cleavage of unstrained cycloketones. With the assistance of the side-chain aryl radical, both cyclohexanones and cyclopentanones are readily cleaved, leading to a variety of structurally diverse 3-

a

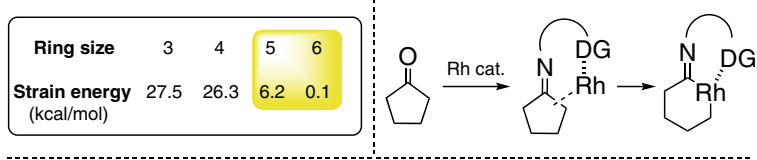

C

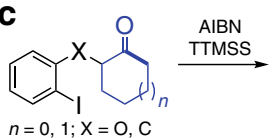<smiles>[X]c1ccccc1OC1CCCCC1</smiles>

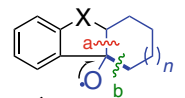
$n=0,1 ; \mathrm{X}=0, \mathrm{C}$<smiles>[X]C1C(=O)c2ccccc2C1=[X]CCCI</smiles>

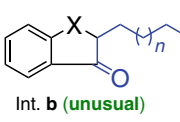

$1^{\circ} \mathrm{C}$-radical $\begin{array}{ll}1^{\circ} C \text {-radical } & 2^{\circ} \text { C-radical, stabilized by adjacen } \\ \text { (active, less stable) } & \text { heteroatom (relatively stable) }\end{array}$

3-coumaran
\& indanone

d
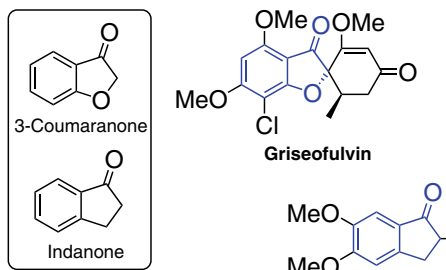

$=0$

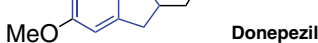

Donepezil

Fig. 1 Challenge and synthetic value for $\mathrm{C}-\mathrm{C}$ cleavage of cyclohexanone and cyclopentanone. a Ring strain energy. $\mathbf{b}$ Activation of cyclopentanone by temporary directing mode by Dong et al. c Aryl radical-promoted activation of both cyclohexanone and cyclopentanone. $\mathbf{d}$ Natural products and drugs containing 3-coumaranone and indane. coumaranones and indanones (Fig. 1c). Notably, these structural motifs are widely found in natural products and pharmaceuticals, manifesting the synthetic value of this protocol (Fig. 1d). Mechanistically, the cleavage of $\mathrm{C}-\mathrm{C} \sigma$-bond via path $\mathbf{b}$ to form the primary alkyl radical (Int. b) is unusual ${ }^{36,37}$, as the generation of ring-expansion Int. a via path $\mathbf{a}$ is supposed to be more favored due to the thermodynamically stability of secondary alkyl radical and $p-\pi$ conjugate effect provided by the adjacent oxygen atom according to the Dowd-Beckwith ring-expansion reaction ${ }^{38-47}$. Thus, density functional theory (DFT) as well as experimental studies have been sought to address the perplexing regioselectivity of $\mathrm{C}-\mathrm{C}$ bond cleavage.

\section{Results}

Reaction parameters survey. The project commenced with an extensive survey of reaction parameters for the $\mathrm{C}-\mathrm{C}$ activation of 2-(2-iodophenoxy) cyclohexanone 1a under radical conditions (Table 1). It was found that with $\mathrm{PhCF}_{3}$ as solvent the combination of AIBN and TTMSS could abstract the iodine atom to generate aryl radical that triggered the subsequent $\mathrm{C}-\mathrm{C}$ cleavage, affording 3-coumaranone $\mathbf{2 a}$ in modest yield (Table 1, entry 1). An alternative pathway, the addition of aryl radical to cyclohexanone followed by dehydration, led to the benzofuran $\mathbf{2} \mathbf{a}^{\prime}$ that was identified as main byproduct in the reaction. While assessing the effect of base, it was surprising that the hydrated $\mathrm{K}_{2} \mathrm{HPO}_{4}$ delivered a good yield (Table 1, entry 2 ), whereas the anhydrous one as well as other bases only afforded a trace amount of products (Table 1, entries 3-4). This prompted us to turn our attention to examining the influence of water (Table 1, entries

Table 1 Reaction parameters survey.

\begin{tabular}{|c|c|c|c|}
\hline & Solvent & Byproduct 2a' & $\begin{array}{l}\text { TMS } \\
\text { TMS-Si-H } \\
\text { TMS } \\
\text { TTMSS }\end{array}$ \\
\hline ntry ${ }^{a}$ & Additive & Solvent & $\begin{array}{l}\text { Yield of } 2 a \\
(\%)^{b}\end{array}$ \\
\hline & None & $\mathrm{PhCF}_{3}$ & 66 \\
\hline & $\begin{array}{l}\mathrm{K}_{2} \mathrm{HPO}_{4} \cdot 3 \mathrm{H}_{2} \mathrm{O}(1 \\
\text { equiv.) }\end{array}$ & $\mathrm{PhCF}_{3}$ & 76 \\
\hline & $\mathrm{K}_{2} \mathrm{HPO}_{4}$ (1 equiv.) & $\mathrm{PhCF}_{3}$ & $<10$ \\
\hline & $\begin{array}{l}\mathrm{K}_{2} \mathrm{CO}_{3} \text { or } \mathrm{K}_{3} \mathrm{PO}_{4}(1 \\
\text { equiv.) }\end{array}$ & $\mathrm{PhCF}_{3}$ & $<10$ \\
\hline & $\mathrm{H}_{2} \mathrm{O}$ (3 equiv.) & $\mathrm{PhCF}_{3}$ & 77 \\
\hline & $\mathrm{H}_{2} \mathrm{O}$ (20 equiv.) & $\mathrm{PhCF}_{3}$ & 85 \\
\hline & $\mathrm{H}_{2} \mathrm{O}$ (100 equiv.) & $\mathrm{PhCF}_{3}$ & 75 \\
\hline & $\mathrm{H}_{2} \mathrm{O}$ (20 equiv.) & DMF & 25 \\
\hline & $\mathrm{H}_{2} \mathrm{O}$ (20 equiv.) & DMSO & 16 \\
\hline 0 & $\mathrm{H}_{2} \mathrm{O}$ (20 equiv.) & THF & 26 \\
\hline 1 & $\mathrm{H}_{2} \mathrm{O}$ (20 equiv.) & DCE & 61 \\
\hline 2 & $\mathrm{H}_{2} \mathrm{O}$ (20 equiv.) & Dioxane & 65 \\
\hline $3^{c}$ & $\mathrm{H}_{2} \mathrm{O}$ (20 equiv.) & $\mathrm{PhCF}_{3}$ & 84 \\
\hline $4^{d}$ & $\mathrm{H}_{2} \mathrm{O}$ (20 equiv.) & $\mathrm{PhCF}_{3}$ & 66 \\
\hline $5^{c, e}$ & $\mathrm{H}_{2} \mathrm{O}$ (20 equiv.) & $\mathrm{PhCF}_{3}$ & 74 \\
\hline $6^{c, f}$ & $\mathrm{H}_{2} \mathrm{O}$ (2O equiv.) & $\mathrm{PhCF}_{3}$ & 56 \\
\hline 78 & None & $\mathrm{PhCF}_{3}$ & 78 \\
\hline $8^{c, g}$ & None & $\mathrm{PhCF}_{3}$ & 31 \\
\hline
\end{tabular}

a Reaction conditions: 1a $(0.2 \mathrm{mmol})$, TTMSS ( $0.3 \mathrm{mmol}, 1.5$ equiv.), AIBN $(0.04 \mathrm{mmol}, 20 \mathrm{~mol} \%)$ and additive in solvent $(2 \mathrm{~mL})$ at $80^{\circ} \mathrm{C}$

bYield of isolated product.

CAIBN $(0.01 \mathrm{mmol}, 5 \mathrm{~mol} \%$ ).

dUse of air instead of AIBN.

${ }^{\mathrm{e}} 70^{\circ} \mathrm{C}$.

fPhCF3 (4 mL).

gBu3SnH instead of TTMSS 


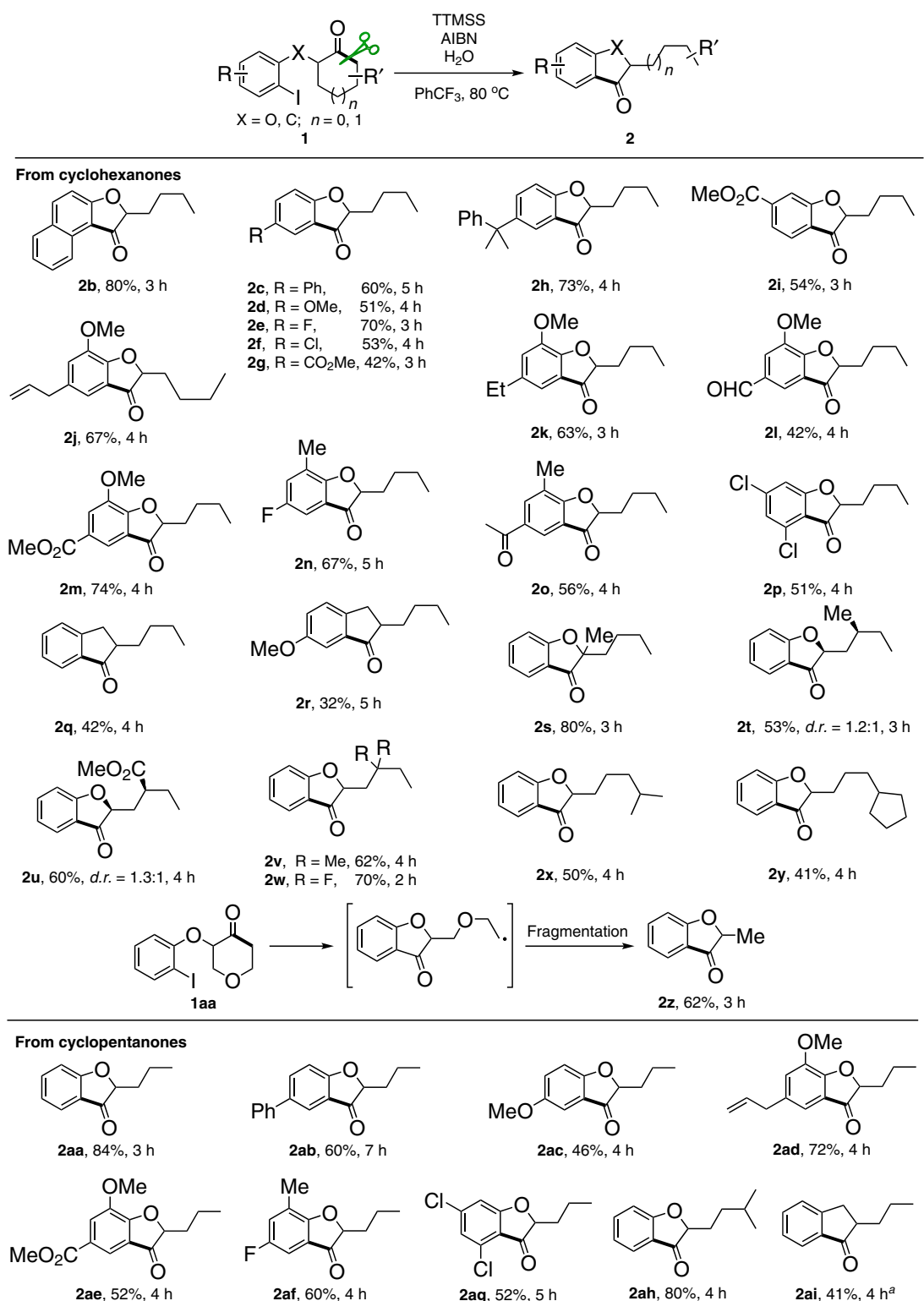

Fig. 2 Generality of protocol and scope of 3-coumaranone and indanone. Reaction conditions: 1 ( 0.2 mmol), TTMSS (0.3 mmol, 1.5 equiv.), AIBN $(0.01 \mathrm{mmol}, 5 \mathrm{~mol} \%)$, and $\mathrm{H}_{2} \mathrm{O}\left(4 \mathrm{mmol}, 20\right.$ equiv.) in $\mathrm{PhCF}_{3}(2 \mathrm{~mL})$ at $80^{\circ} \mathrm{C}$. Yields of isolated products are given. ${ }^{\text {a }}$ 1ai $(0.3 \mathrm{mmol}), \mathrm{TTMSS}(1.5$ equiv.), $\mathrm{V}-40\left(20 \mathrm{~mol} \%\right.$ ), and $\mathrm{K}_{2} \mathrm{HPO}_{4} \cdot 3 \mathrm{H}_{2} \mathrm{O}$ (1 equiv.) in $\mathrm{PhCF}_{3}(6 \mathrm{~mL})$ at $110{ }^{\circ} \mathrm{C}$.

$5-7)$. Indeed, the addition of 20 equiv. of water increased the yield to $85 \%$ (Table 1 , entry 6). Solvent screening revealed that $\mathrm{PhCF}_{3}$ was more efficient than other solvents (Table 1, entries 8-12). Reducing the loading of AIBN to $5 \mathrm{~mol} \%$ resulted in a similarly high yield (Table 1, entry 13). Notably, the reaction could be carried out under air without the use of AIBN, leading to a synthetically useful yield (Table 1, entry 14). Decrease of reaction temperature compromised the reaction outcome (Table 1, entry 15). Reducing the concentration also decreased the reaction yield (Table 1, entry 16). Although using $\mathrm{Bu}_{3} \mathrm{SnH}$ instead of TTMSS also led to good yield (Table 1, entry 17, with $20 \mathrm{~mol} \%$ of AIBN; Table 1 , entry 18 , with $5 \mathrm{~mol} \%$ of AIBN), the use of less toxic silane is still preferred in the reaction.

Scope of substrates. With the optimized reaction conditions in hand, we set about evaluating the generality of the protocol (Fig. 2). A variety of 2-substituted cyclohexanones (1b-1z) were firstly examined. All the reactions were readily accomplished through the regiospecific cyclic $\mathrm{C}-\mathrm{C} \sigma$-bond scission and subsequent $\mathrm{C}-\mathrm{C}$ bond formation within a few hours. Both electron-rich and -deficient aryl substituents were well tolerated in the reaction, affording the corresponding 3-coumaranones 2 . Under the identical conditions, the naphthyl-fused coumaranone 2b was also obtained in a high yield. The protocol could furnish a diversity of multiple functionalized 3-coumaranones $(\mathbf{2} \mathbf{j}-\mathbf{2} \mathbf{p})$ even bearing susceptible groups such as allyl (2j) and formyl (2l). Indanones $(\mathbf{2 q}-\mathbf{2 r})$ could be prepared by this method, albeit in relatively lower yields. Notably, the use of 2,2-disubstituted cyclohexanone 1s did not reverse the regioselectivity, though the competitive ring-expansion pathway seemed more likely due to the formation of thermodynamically favored tertiary alkyl radical. Substitutions on the cyclic framework did not influence the transformation, leading to the corresponding 3-coumaranones (2t-2y) with various aliphatic side chains. Interestingly, the reaction with tetrahydropyranone $\mathbf{1 z}$ resulted in 2-methyl-3coumaranone $2 \mathbf{z}$ probably after the fragmentation of the ringopened radical intermediate (see Supplementary Fig. 12). Some representative examples of cyclopentanones (1aa-1ai) were then 
<smiles>O=C1CCCCC1Oc1ccccc1Br</smiles>

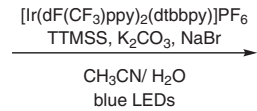<smiles>O=C1CCCC1Oc1ccccc1Br</smiles>

$8 b$

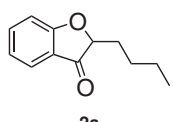

2a

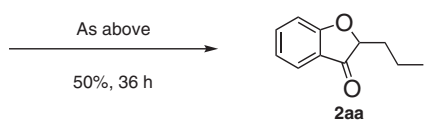

Fig. 3 Transformation of arylbromide analogs. a Reaction with 2-(2 bromophenoxy) cyclohexanone. b Reaction with 2-(2-bromophenoxy) cyclopentanone.

a

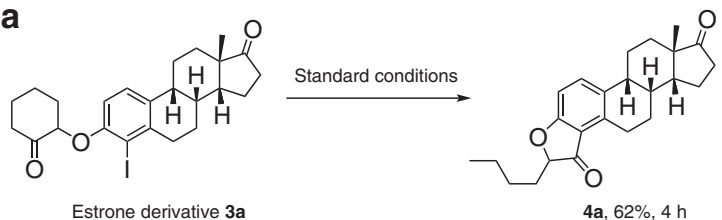

b

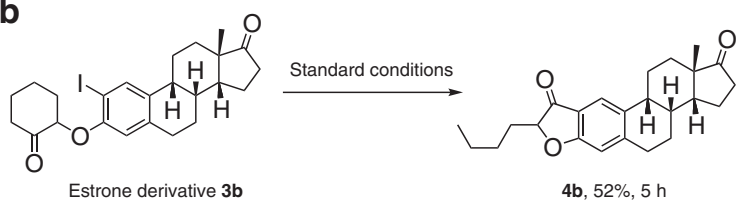

Fig. 4 Modification of complex natural product. a Reaction with Estrone derivative 3a. b Reaction with Estrone derivative $\mathbf{3 b}$.

investigated. The substitution effect on either arene or cyclopentanone was examined. Likewise, the reaction readily proceeded regardless of the electronic and steric characters, affording the resultant 3-coumaranones (2aa-2ah) in synthetically useful yields. The corresponding indanone (2ai) could also be obtained by this method, but with the modified conditions. Notably, ring opening of cycloheptanone could also afford the corresponding product 2aj in 30\% yield (see Supplementary Fig. 13).

The conversion of 2-(2-bromophenoxy) cyclohexanone $\mathbf{8 a}$ and cyclopentanone $\mathbf{8 b}$ could not occur under the current condition due to the lower reactivity of arylbromide. However, the expected transformation proceeded with a modified photochemical condition, affording the corresponding products in synthetically useful yields (Fig. 3).

The practicality of the method was further illustrated in the modification of complex natural products (Fig. 4). Despite the location of iodine atom, both the estrone derivative $\mathbf{3 a}$ and $\mathbf{3 b}$ were readily converted to the corresponding coumaranones $4 \mathbf{a}$ and $\mathbf{4 b}$ in synthetically useful yields, respectively.

Mechanistic studies. To shed light on the unusual regioselectivity of the cyclic $\mathrm{C}-\mathrm{C}$ cleavage, we conducted computational studies using DFT calculations (for details, see the SI). The results are presented in Fig. 5. The cleavage of the $\mathrm{C} 1-\mathrm{C} 2$ bond in Int.1 via TS1a (Fig. 5a, path a) to give the nine-membered ring radical intermediate (Int.a) is a facile and reversible process with a barrier of only $3.2 \mathrm{kcal} / \mathrm{mol}$. The energy barrier is $3.4 \mathrm{kcal} / \mathrm{mol}$ higher for the cleavage of the C1-C3 bond (via TS1b, path b) to generate the primary alkyl radical intermediate Int.b. As shown in Fig. 5b, the natural bond orbital (NBO) analysis of Int.1 reveals the hyperconjugation between the lone pairs at $\mathrm{O} 4\left(n_{\mathrm{O} 4}\right)$ and the antibonding orbitals of the $\mathrm{C} 1-\mathrm{C} 3$ and $\mathrm{C} 1-\mathrm{C} 2$ bonds $\left(\sigma^{*} \mathrm{C} 1-\mathrm{C} 3\right.$ and $\sigma^{*} \mathrm{C} 1-\mathrm{C} 2$ ), which would weaken both $\mathrm{C}-\mathrm{C}$ single bonds and thus facilitate their cleavage ${ }^{48}$. The $n_{\mathrm{O} 4} \rightarrow \sigma^{*} \mathrm{C} 1-\mathrm{C} 2$ interaction was calculated to be about $7 \mathrm{kcal} / \mathrm{mol}$ stronger than the $n_{\mathrm{O} 4} \rightarrow \sigma^{*}$ C1-C3 interaction (see Supplementary Tables 1 and 2). This may explain why the cleavage of the $\mathrm{C} 1-\mathrm{C} 2$ bond exhibits a lower energy barrier.

Interestingly, Int.a is $1.8 \mathrm{kcal} / \mathrm{mol}$ less stable than Int.b, presumably due to a greater ring strain in the nine-membered ring. The subsequent hydrogen-atom abstraction is irreversible and requires a higher barrier than the cleavage of $\mathrm{C}-\mathrm{C}$ bond. The barriers for hydrogen-atom abstraction from TTMSS by Int.a and Int.b are 15.2 (via TS2a) and 10.9 (via TS2b) $\mathrm{kcal} / \mathrm{mol}$, respectively, which are at least $4.3 \mathrm{kcal} / \mathrm{mol}$ higher than that for the cyclic $\mathrm{C}-\mathrm{C}$ cleavage. The formation of 3-coumaranone $\mathbf{2 a}$ is thermodynamically favored over 3,4,5,6-tetrahydrobenzo[b]oxonin-7 $(2 \mathrm{H})$-one $2 \mathbf{a}^{\prime}$ by $12.8 \mathrm{kcal} / \mathrm{mol}$. Accordingly, the 3coumaranone $\mathbf{2 a}$ is both kinetically and thermodynamically favored product, which is consistent with experimental observation that only 2a was observed. Notably, an intramolecular hydrogen-atom abstraction (1,5-H transfer) is also possible for Int.b. The calculations show that the intramolecular 1,5hydrogen atom abstraction proceeds via TS3b has a barrier of $10.4 \mathrm{kcal} / \mathrm{mol}$, leading to a tertiary carbon radical intermediate Int.2, which is quite stable and located $26.2 \mathrm{kcal} / \mathrm{mol}$ below Int.1. Int.2 abstracts a hydrogen atom from TTMSS to deliver the final product via TS4b with a barrier of $19.8 \mathrm{kcal} / \mathrm{mol}$. Since the intramolecular 1,5-hydrogen atom abstraction is slightly favored (only $0.5 \mathrm{kcal} / \mathrm{mol}$ ) over the intermolecular hydrogen-atom abstraction (TS2b vs. TS3b), the two processes could compete with each other. This corresponds well with the isotope-labeling experiments (Fig. 6). It could also be speculated that Int.1, Int.a, and Int.b are in an equilibrium and a high energy barrier for $\mathrm{H}$ transfer from TTMSS for cyclic radical Int.a may allow for preferential quenching of primary alkyl radical Int.b via either intramolecular 1,5-H-shift or intermolecular $\mathrm{H}$-shift from TTMSS. It may be possible for alkoxy radical Int.1 to abstract hydrogen from TTMSS to form alcohol. Dehydration of the product can give the benzofuran-type byproduct. Overall, the calculations revealed that the selectivity of the reaction is mainly controlled by the step of hydrogen-atom abstraction instead of the $\mathrm{C}-\mathrm{C}$ bond cleavage. The primary alkyl radical is more reactive than the secondary alkyl radical for hydrogen atom abstraction, leading to the observed selectivity. Replacing TTMSS by tin hydride as hydrogen atom donor leads to the similar calculational outcome (see Supplementary Fig. 178). The rational analysis for the formation of indanones could also be obtained from the DFT studies (see Supplementary Fig. 179).

To provide support for the computational results, we further carried out the experiments with the deuterium-labeled materials. Firstly, the reaction of $\mathbf{1 a}$ in the presence of $d$-TTMSS delivered product 5 with $\mathrm{H} / \mathrm{D}$ ratio as $0.7: 0.3$, indicating that the final step of $\mathrm{H}$-abstraction could proceed via either the intramolecular 1,5HAT or intermolecular H-abstraction from TTMSS (Fig. 6a). While employing the deuterated cyclohexanone 6 under the previous conditions, the counting of $\mathrm{H}$ and $\mathrm{D}$ in product 7 also suggested that the radical chain termination step could go through both two pathways (Fig. 6b).

A further attempt was conducted to elucidate the effect of water (for details, see Supplementary Figs. 8-10). After the completion of reaction, a highly acidic water drop $(\mathrm{pH}<1$, presumably the aqueous solution of $\mathrm{HI}$ ) precipitated in the reaction vial, which was obtained by hydrolysis of the byproduct (TMS) ${ }_{3} \mathrm{SiI}^{49,50}$. Moreover, the addition of TFA instead of water to the reaction largely suppressed the reaction outcome by increasing the formation of benzofuran byproduct $\mathbf{2} \mathbf{a}^{\prime}$. Thus, it was hypothesized that the HI-induced acidic condition was fatal to the reaction, and water could separate $\mathrm{HI}$ from the reaction through the formation of a biphasic solution. 
a

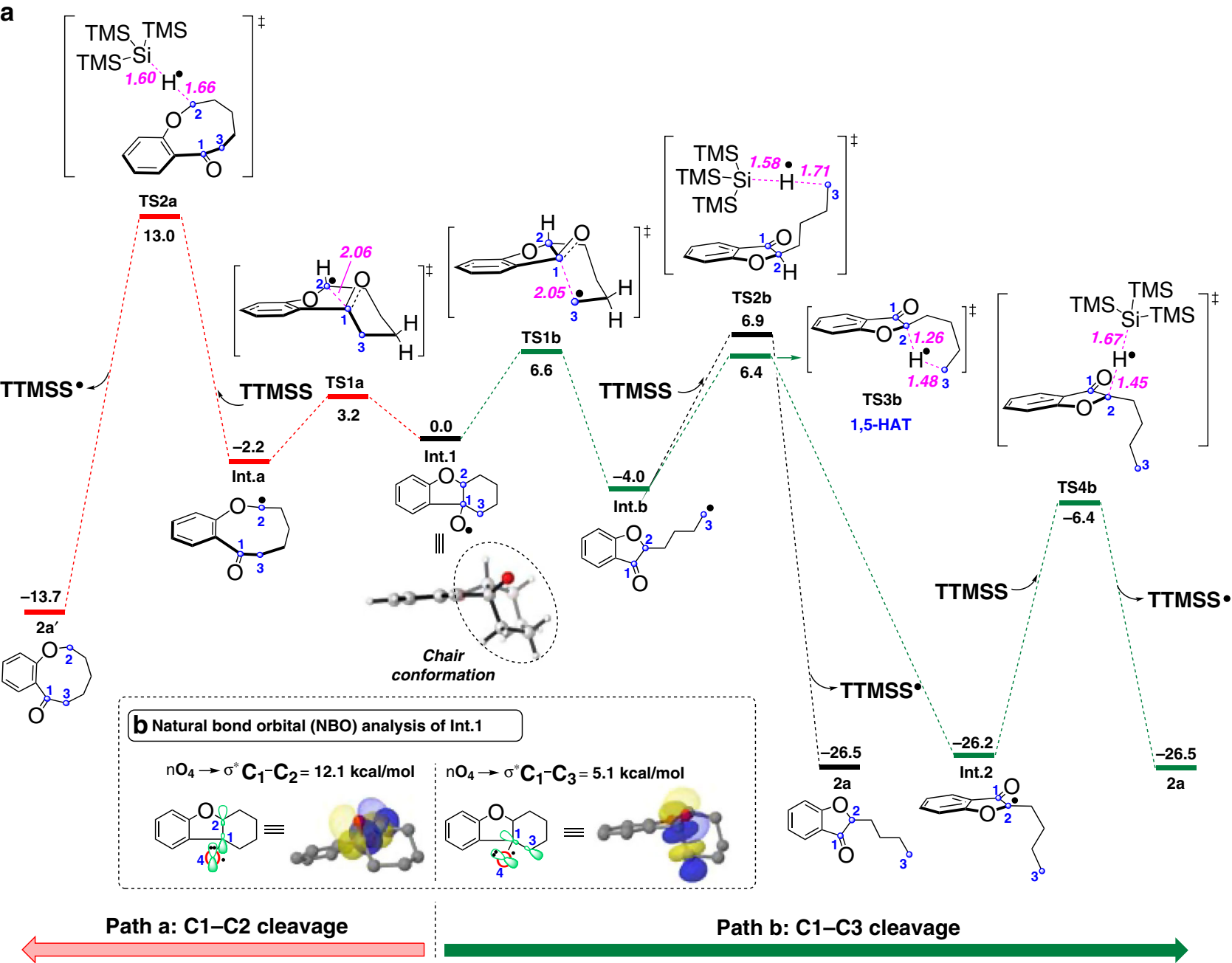

Fig. 5 Computational study. a Free energy diagram (kcal/mol) calculated at the (SMD)-M06-2×/6-311++G(2d,p)//M06-2×/6-31G(d) level of theory (pink: bond length in $\AA$ ). $\Delta G$ calculated at $298.15 \mathrm{~K}$. b The natural bond orbital (NBO) analysis of Int.1. Interaction energies are in kcal/mol.

a<smiles>O=C1CCCCC1Oc1ccccc1I</smiles>

1a
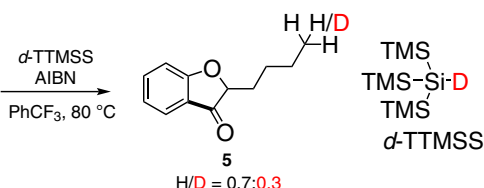

b

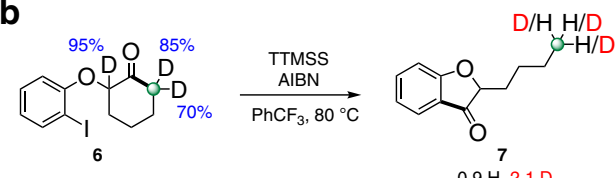

Fig. 6 Deuterium labeling experiments. a Reaction with d-TTMSS.

b Reaction with deuterated cyclohexanone 6 .

\section{Discussion}

In summary, we have disclosed an efficient radical-mediated protocol for the cleavage of inert unstrained $\mathrm{C}-\mathrm{C} \sigma$-bonds. Both cyclohexanones and cyclopentanones prove to be suitable substrates. A variety of synthetically valuable 3-coumaranones or indanones are readily prepared under mild conditions. The cyclic $\mathrm{C}-\mathrm{C}$ bonds are cleaved in an unusual regioselectivity. DFT studies as well as the deuterium labeling experiments are sought to address the selectivity issue. This protocol provides a practical method and informative mechanistic insight for the elusive $\mathrm{C}-\mathrm{C}$ activation of unstrained cycloketones.

\section{Methods}

General procedure for ring opening of cycloketones. Cycloketones (1) $(0.2 \mathrm{mmol}, 1$ equiv. $)$ and AIBN $(0.01 \mathrm{mmol}, 5 \mathrm{~mol} \%)$ were loaded in a reaction vial that was subjected to evacuation/flushing with $\mathrm{N}_{2}$ three times. Then TTMSS ( $0.3 \mathrm{mmol}, 1.5$ equiv.), $\mathrm{H}_{2} \mathrm{O}$ (4 mmol, 20 equiv.) and $\mathrm{PhCF}_{3}(2 \mathrm{~mL})$ were added to the mixture which was then heated to $80^{\circ} \mathrm{C}$. After the reaction completion, the reaction mixture was concentrated in vacuo. Purification by flash column chromatography on silica gel afforded the desired product.

\section{Data availability}

The authors declare that all other data supporting the findings of this study are available within the article and Supplementary Information files, and also are available from the corresponding author on reasonable request.

Received: 8 August 2019; Accepted: 8 January 2020; Published online: 03 February 2020

\section{References}

1. Jun, C.-H. Transition metal-catalyzed carbon-carbon bond activation. Chem. Soc. Rev. 33, 610-618 (2004).

2. Tobisu, M. \& Chatani, N. Catalytic reactions involving the cleavage of carboncyano and carbon-carbon triple bonds. Chem. Soc. Rev. 37, 300-307 (2008).

3. Dong, G. B. C-C Bond Activation, Vol. 346. Topics in Current Chemistry (Springer, Berlin, 2014).

4. Dermenci, A., Coe, J. W. \& Dong, G. B. Direct activation of relatively unstrained carbon-carbon bonds in homogeneous systems. Org. Chem. Front. 1, 567-581 (2014) 
5. Chen, F., Wang, T. \& Jiao, N. Recent advances in transition-metal-catalyzed functionalization of unstrained carbon-carbon bonds. Chem. Rev. 114 8613-8661 (2014).

6. Murakami, M. \& Ishida, N. Potential of metal-catalyzed C-C single bond cleavage for organic synthesis. J. Am. Chem. Soc. 138, 13759-13769 (2016).

7. Kim, D.-S., Park, W.-J. \& Jun, C.-H. Metal-organic cooperative catalysis in C-H and C-C bond activation. Chem. Rev. 117, 8977-9015 (2017).

8. Liu, H., Feng, M. \& Jiang, X. Unstrained carbon-carbon bond cleavage. Chem. Asian J. 9, 3321-3649 (2014).

9. Song, F., Gou, T., Wang, B.-Q. \& Shi, Z.-J. Catalytic activations of unstrained C-C bond involving organometallic intermediates. Chem. Soc. Rev. 47, 7078-7115 (2018).

10. Rybtchinski, B. B. \& Milstein, D. Metal insertion into C-C bonds in solution. Angew. Chem. Int. Ed. 38, 870-883 (1999).

11. Sadan, A. K., Saini, R. K. \& Billups, W. E. Cyclobutarenes and related compounds. Chem. Rev. 103, 1539-1602 (2003).

12. Rubin, M., Rubina, M. \& Gevorgyan, V. Transition metal chemistry of cyclopropenes and cyclopropanes. Chem. Rev. 107, 3117-3179 (2007).

13. Carson, C. A. \& Kerr, M. A. Heterocycles from cyclopropanes: applications in natural product synthesis. Chem. Soc. Rev. 38, 3051-3060 (2009).

14. Seiser, T. \& Cramer, N. Enantioselective metal-catalyzed activation of strained rings. Org. Biomol. Chem., 7, 2835-2840 (2009).

15. Seiser, T., Saget, T., Tran, D. N. \& Cramer, N. Cyclobutanes in catalysis. Angew. Chem. Int. Ed. 50, 7740-7752 (2011).

16. Flores-Gaspar, A. \& Martin, R. Recent advances in the synthesis and application of benzocyclobutenones and related compounds. Synthesis $\mathbf{4 5}$, 563-580 (2013).

17. Souillart, L. \& Cramer, N. Catalytic C-C bond activations via oxidative addition to transition metals. Chem. Rev. 115, 9410-9464 (2015).

18. Marek, I., Masarwa, A., Delaye, P. O. \& Leibeling, M. Selective carbon-carbon bond cleavage for the stereoselective synthesis of acyclic systems. Angew. Chem. Int. Ed. 54, 414-429 (2015).

19. Chen, P.-H. \& Dong, G. B. Cyclobutenones and benzocyclobutenones: versatile synthons in organic synthesis. Chem. Eur. J. 22, 18290-18315 (2016).

20. Fumagalli, G., Stanton, S. \& Bower, J. F. Recent methodologies that exploit $\mathrm{C}-\mathrm{C}$ single-bond cleavage of strained ring systems by transition metal complexes. Chem. Rev. 117, 9404-9432 (2017).

21. Ren, R. G. \& Zhu, C. Radical-mediated ring-opening functionalization of cyclobutanols: a shortcut to $\gamma$-substituted ketones. Synlett 27, 1139-1144 (2016).

22. $\mathrm{Wu}, \mathrm{X} . \mathrm{X} . \& \mathrm{Zhu}, \mathrm{C}$. Recent advances in ring-opening functionalization of cycloalkanols by C-C o-bond cleavage. Chem. Rec. 18, 587-598 (2018)

23. Wang, Y.-F. \& Chiba, S. Mn(III)-mediated reactions of cyclopropanols with vinyl azides: synthesis of pyridine and 2-azabicyclo[3.3.1]non-2-en-1-ol derivatives. J. Am. Chem. Soc. 131, 12570-12572 (2009).

24. Zhao, H. J., Fan, X. F., Yu, J. J. \& Zhu, C. Silver-catalyzed ring-opening strategy for the synthesis of $\beta$ - and $\gamma$-fluorinated ketones. J. Am. Chem. Soc. 137, 3490-3493 (2015).

25. Jia, K. F., Zhang, F. Y., Huang, H. C. \& Chen, Y. Y. Visible-light-induced alkoxyl radical generation enables selective $\mathrm{C}\left(\mathrm{sp}^{3}\right)-\mathrm{C}\left(\mathrm{sp}^{3}\right)$ bond cleavage and functionalizations. J. Am. Chem. Soc. 138, 1514-1517 (2016).

26. Ren, R. G., Zhao, H. J., Huan, L. T. \& Zhu, C. Manganese-catalyzed oxidative azidation of cyclobutanols: regiospecific synthesis of alkyl azides by $\mathrm{C}-\mathrm{C}$ bond cleavage. Angew. Chem. Int. Ed. 54, 12692-12696 (2015).

27. Ren, R. G., Wu, Z., Xu, Y. \& Zhu, C. C-C bond-forming strategy by manganese-catalyzed oxidative ring-opening cyanation and ethynylation of cyclobutanol derivatives. Angew. Chem. Int. Ed. 55, 2866-2869 (2016).

28. Yayla, H. G., Wang, H. J., Tarantino, K. T., Orbe, H. S. \& Knowles, R. R. Catalytic ring-opening of cyclic alcohols enabled by PCET activation of strong O-H bonds. J. Am. Chem. Soc. 138, 10794-10797 (2016).

29. Guo, J.-J. et al. Photocatalytic C-C bond cleavage and amination of cycloalkanols by cerium(III) chloride complex. Angew. Chem. Int. Ed. 55, 15319-15322 (2016).

30. Fan, X. F., Zhao, H. J., Yu, J. J., Bao, X. G. \& Zhu, C. Regiospecific synthesis of distally chlorinated ketones via C-C bond cleavage of cycloalkanols. Org. Chem. Front. 3, 227-232 (2016).

31. Wang, D. P., Mao, J. C. \& Zhu, C. Visible light-promoted ring-opening functionalization of unstrained cycloalkanols via inert $\mathrm{C}-\mathrm{C}$ bond scission. Chem. Sci. 9, 5805-5809 (2018).

32. Khoury, P. R., Goddard, J. D. \& Tam, W. Ring strain energies: substituted rings, norbornanes, norbornenes and norbornadienes. Tetrahedron $\mathbf{6 0}$, 8103-8112 (2004).

33. Xia, Y., Lu, G., Liu, P. \& Dong, G. B. Catalytic activation of carbon-carbon bonds in cyclopentanones. Nature 539, 546-550 (2016).

34. Xia, Y., Wang, J. B. \& Dong, G. B. Distal-bond-selective C-C activation of ring-fused cyclopentanones: an efficient access to spiroindanones. Angew. Chem. Int. Ed. 56, 2376-2380 (2017).
35. Xia, Y., Wang, J. B. \& Dong, G. B. Suzuki-Miyaura coupling of simple ketones via activation of unstrained carbon-carbon bonds. J. Am. Chem. Soc. 140, 5347-5351 (2018).

36. Macdonald, T. L. \& O'Dell, D. E. Beta-scission of 9-decalinoxyl and related free radicals. J. Org. Chem. 46, 1501-1503 (1981).

37. Beckwith, A. L. J., Kazlauskas, R. \& Syner-Lyons, M. R. Beta-fission of 9decalinoxyl radicals: reversible formation of 6-ketocyclodecyl radical. J. Org. Chem. 48, 4718-4722 (1983).

38. Dowd, P. \& Choi, S. C. A new tributyltin hydride-based rearrangement of bromomethyl beta-keto esters. a synthetically useful ring expansion to gamma-keto esters. J. Am. Chem. Soc. 109, 3493-3494 (1987).

39. Dowd, P. \& Choi, S. C. Free radical ring expansion by three and four carbons. J. Am. Chem. Soc. 109, 6548-6549 (1987).

40. Beckwith, A. L. J., O’Shea, D. M. \& Westwood, S. W. Rearrangement of suitably constituted aryl, alkyl, or vinyl radicals by acyl or cyano group migration. J. Am. Chem. Soc. 110, 2565-2575 (1988).

41. Dowd, P. \& Zhang, W. Free radical-mediated ring expansion and related annulations. Chem. Rev. 93, 2091-2115 (1993).

42. Beckwith, A. L. J. \& Hay, B. P. Kinetics and mechanism of the exo cyclizations of omega-formylalkyl radicals. J. Am. Chem. Soc. 111, 2674-2681 (1989).

43. Walton, R. \& Fraser-Reid, B. Studies on the intramolecular competitive addition of carbon radicals to aldehydo and alkenyl groups. J. Am. Chem. Soc. 113, 5791-5799 (1991).

44. Scheer, A. M., Welz, O., Sasaki, D. Y., Osborn, D. L. \& Taatjes, C. A. Facile rearrangement of 3-oxoalkyl radicals is evident in low-temperature gas-phase oxidation of ketones. J. Am. Chem. Soc. 135, 14256-14265 (2013).

45. Hierold, J. \& Lupton, D. W. Synthesis of spirocyclic $\gamma$-lactones by cascade beckwith-dowd ring expansion/cyclization. Org. Lett. 14, 3412-3415 (2012).

46. Baroudi, A., Mauldin, J. \& Alabugin, I. V. Conformationally gated fragmentations and rearrangements promoted by interception of the bergman cyclization through intramolecular $\mathrm{H}$-abstraction: a possible mechanism of auto-resistance to natural enediyne antibiotics? J. Am. Chem. Soc. 132, 967-979 (2010)

47. Harris, T., Gomes, Gd. P., Clark, R. J. \& Alabugin, I. V. Domino fragmentations in traceless directing groups of radical cascades: evidence for the formation of alkoxy radicals via C-O scission. J. Org. Chem. 81, 6007-6017 (2016)

48. Alabugin, I. V. Stereoelectronic Effects: A Bridge Between Structure and Reactivity (Wiley, Hoboken, 2016).

49. Damja, R. I. \& Eaborn, C. Preparation and reactions of some sterically hindered silanols, including [tris(trimethylsilyl)methyl] silanetriol. J. Organomet. Chem. 290, 267-275 (1985).

50. Zhilitskaya, L. V., Yarosh, N. O., Shagun, L. G., Dorofeev, I. A. \& Larina, L. I. Siloxane derivatives of 2-mercaptobenzothiazole. Mendeleev Commun. 27, 352-354 (2017).

\section{Acknowledgements}

We are grateful for the financial support from the National Natural Science Foundation of China (21722205, 21971173, 21772098, and 21933004), the Project of Scientific and Technologic Infrastructure of Suzhou (SZS201708), and the Priority Academic Program Development of Jiangsu Higher Education Institutions (PAPD).

\section{Author contributions}

C.Z. conceived and designed the experiments; M.W. and S.Y. carried out most of the experiments; M.L. and X.-S.X carried out DFT computation; M.W., M.L., S.Y., X.-S.X., X.W., and C.Z. analyzed data; X.-S.X. and C.Z. wrote the paper; C.Z. directed the project.

\section{Competing interests}

The authors declare no competing interests.

\section{Additional information}

Supplementary information is available for this paper at https://doi.org/10.1038/s41467 020-14435-5.

Correspondence and requests for materials should be addressed to X.-S.X. or C.Z.

Peer review information Nature Communications thanks Wen-Jie Chen, Xin-Hua Duan and the other, anonymous, reviewer(s) for their contribution to the peer review of this work. Peer reviewer reports are available.

Reprints and permission information is available at http://www.nature.com/reprints

Publisher's note Springer Nature remains neutral with regard to jurisdictional claims in published maps and institutional affiliations. 
(c) (i) Open Access This article is licensed under a Creative Commons Attribution 4.0 International License, which permits use, sharing, adaptation, distribution and reproduction in any medium or format, as long as you give appropriate credit to the original author(s) and the source, provide a link to the Creative Commons license, and indicate if changes were made. The images or other third party material in this article are included in the article's Creative Commons license, unless indicated otherwise in a credit line to the material. If material is not included in the article's Creative Commons license and your intended use is not permitted by statutory regulation or exceeds the permitted use, you will need to obtain permission directly from the copyright holder. To view a copy of this license, visit http://creativecommons.org/ licenses/by/4.0/.

(C) The Author(s) 2020 